\title{
Magnetospheric accretion \& outflows in stars \& brown dwarfs: theories and observational constraints
}

\author{
S. Mohanty ${ }^{1}$ \\ ${ }^{1}$ Imperial College, London
}

The manner in which young classical T Tauri stars (cTTs) and brown dwarfs accrete gas from their surrounding disks and simultaneously drive jets and outflows is central to star and planet formation and angular momentum evolution, but remains an ill-understood and hotly debated subject. One of the central concerns is the stellar field geometry: while analytic theories assume an idealized stellar dipole, T Tauri fields are observed to be complex multipolar beasts. I present an analytic generalization of the $\mathrm{X}$-wind theory to include such fields. Independent of the precise field geometry, the generalized model makes a unique prediction about the relationship between various cTTs observables. I show that this prediction is supported by observations of accretion rate, hot spot size, stellar rotation and field strength from stellar to brown dwarf masses, including recent detailed spectropolarimetric measurements. I also discuss the unique insights offered by recent magnetic field measurements on accreting brown dwarfs: while they agree with the accretion theory above, they also pose a puzzle for magnetic field generation theory. Resolving this conundrum promises to illuminate our general picture of accretion and angular momentum transport in fully convective objects.

Editors Note: More details on this work have been published by Mohanty 85 Shu (2008).

\section{References}

Mohanty, S. \& Shu, F. H. 2008, ApJ 687, 1323 